\title{
The Effects of Stratification on Flow Separation
}

\author{
MaArten H. P. Ambaum And David P. Marshall \\ Department of Meteorology, University of Reading, Reading, United Kingdom
}

(Manuscript received 16 June 2004, in final form 2 December 2004)

\begin{abstract}
Separation of stratified flow over a two-dimensional hill is inhibited or facilitated by acceleration or deceleration of the flow just outside the attached boundary layer. In this note, an expression is derived for this acceleration or deceleration in terms of streamline curvature and stratification. The expression is valid for linear as well as nonlinear deformation of the flow. For hills of vanishing aspect ratio a linear theory can be derived and a full regime diagram for separation can be constructed. For hills of finite aspect ratio scaling relationships can be derived that indicate the presence of a critical aspect ratio, proportional to the stratification, above which separation will occur as well as a second critical aspect ratio above which separation will always occur irrespective of stratification.
\end{abstract}

\section{Introduction}

A key aspect of stratified flow over hills that remains only partially understood is the separation of the stably stratified boundary layer. Numerical simulations (e.g., Mason 1987), laboratory experiments (e.g., Baines and Hoinka 1985), and observations (e.g., Holden et al. 2000) of quasi-two-dimensional flow over elongated hills reveal a range of separation regimes dependent on the nondimensional parameters $N h / U$ and $h / L$ (the aspect ratio), where $N$ is the buoyancy frequency, $h$ is the height of the hill, $U$ is the upstream velocity, and $L$ is the half-width of the hill. For low $N h / U$ and low $h / L$ no separation will occur. In the limit of weak stratification $(N h / U<1)$, the boundary layer separates on the lee of a hill if the aspect ratio is high enough. However in the limit of strong stratification $(N h / U>1)$, downstream separation is either delayed or completely suppressed while blocked flow is observed on the windward side. For large enough $N h / U$ attached strong downslope flows are obtained, although postwave separation may occur. For short hills (high aspect ratio) leeside separation occurs.

In classical fluid dynamics, boundary layer separation

Corresponding author address: Dr. Maarten Ambaum, Dept. of Meteorology, University of Reading, P.O. Box 243, Reading RG6 6BB, United Kingdom.

E-mail: m.h.p.ambaum@rdg.ac.uk occurs when the nearly inviscid flow just outside the viscous boundary layer undergoes a finite deceleration through an adverse pressure gradient. This results in a singularity in the attached boundary layer that can only be removed if the flow separates (Goldstein 1948; Stewartson 1970). While turbulence or diabatic heating will modify this behavior, the presence, or otherwise, of an adverse pressure gradient remains a good indicator of whether separation is liable to occur (e.g., Doyle and Durran 2002).

There is an analogy between the effects of stratification on flow over an orographic obstacle and the $\beta$ effect of differential rotation on horizontal flow (Long 1953). In the ocean, the $\beta$ effect inhibits separation of eastward flow (analogous to stable stratification) on the lee of a vertical obstacle, with a separated region appearing upstream (Merkine 1980; Boyer and Davies 1982; Foster 1985; Page and Johnson 1990; Tansley and Marshall 2001). Marshall and Tansley (2001) derived an implicit formula to determine the conditions under which a deceleration of the flow, and thus separation, is liable to occur in the ocean. They found that a deceleration is related to three distinct processes: the $\beta$ effect, changes in streamline curvature, and vortex stretching. In particular, they found that the $\beta$ effect always accelerates western boundary currents, consistent with the inhibition of separation on the eastern side of obstacles.

The aim of this note is to derive the analogous implicit formula applicable to stratified flow past a hill. 
This will enable us to determine the conditions under which separation is liable to occur through deceleration of the boundary layer (suitably generalized to account for the effects of stratification). We further apply this formula to linear waves over corrugated orography and show that we can reproduce the separation properties, as a function of $N h / U$ and the aspect ratio $h / L$ of the hill, observed in laboratory studies.

The note is organized as follows. In section 2, the separation condition is derived. In section 3 , the two asymptotic regimes of hills of low aspect ratio and hills of $O(1)$ aspect ratio are analyzed. A brief concluding discussion is given in section 4 .

\section{Separation condition}

\section{a. Model formulation}

The inviscid two-dimensional Euler equations are used to describe the laminar attached flow over a hill. This confines our description to the so-called outer region of the planetary boundary layer (e.g., Belcher and Wood 1996) where the Reynolds-stress gradients can be ignored compared to the inertial terms. The outer region is separated from the surface by an inner region where the turbulence is close to local equilibrium. To a good approximation, the thickness of this inner region can be assumed constant if no separation occurs. The lower boundary of our domain is the top of the inner region. The upstream velocity profile in the outer region is assumed to be in equilibrium and therefore prescribed as an upstream boundary condition. This upstream velocity profile may be thought of as the outer part of the usual log-linear boundary layer profile. In section 3 this profile is approximated with a constant velocity to simplify the analysis. Such a constant profile is also appropriate for typical experimental setups where a ridge is towed into quiescent fluid.

Now consider two-dimensional, steady flow in the $(x$, $z$ ) plane. The equations of motion are

$$
\begin{array}{r}
\mathbf{u} \cdot \nabla u+\frac{p_{x}}{\rho}=0, \\
\mathbf{u} \cdot \nabla w+\frac{p_{z}}{\rho}+g=0, \\
\nabla \cdot(\rho \mathbf{u})=0,
\end{array}
$$

where $\mathbf{u}=(u, w)$ is the fluid velocity, $p$ is pressure, $\rho$ is density, and $g$ is the gravitational acceleration. The flow is assumed to be ideal and adiabatic such that the thermodynamic equation can be written as

$$
\mathbf{u} \cdot \boldsymbol{\nabla} \theta=0
$$

where

$$
\theta=T\left(\frac{p_{0}}{p}\right)^{R / C_{p}}
$$

is the potential temperature for some constant reference pressure $p_{0} ; R$ and $C_{p}$ are the gas constant and specific heat capacity at constant pressure.

Cross-differentiating Eqs. (1) and (2) gives a vorticity equation

$$
\boldsymbol{\nabla} \cdot(\zeta \mathbf{u})+J\left(\frac{1}{\rho}, p\right)=0,
$$

where $\zeta=w_{x}-u_{z}$ is the vorticity and $J(a, b)=a_{x} b_{z}-$ $a_{z} b_{x}$ is the Jacobian of $a$ and $b .{ }^{1}$ Using the equation of state, the thermodynamic equation and the ideal gas law, $p=\rho R T$, the solenoidal term in the vorticity equation can be rewritten as

$$
J\left(\frac{1}{\rho}, p\right)=\frac{J(\theta, p)}{\rho \theta}=-\frac{N^{2}}{g \rho u} \mathbf{u} \cdot \nabla p,
$$

with $N^{2}=g \theta_{z} / \theta$ the local buoyancy frequency. In the above equation, $\mathbf{u} \cdot \boldsymbol{\nabla} p$ may be evaluated using the momentum equations to arrive at the following vorticity equation:

$$
\nabla \cdot(\zeta \mathbf{u})+\frac{N^{2}}{g u} \mathbf{u} \cdot \nabla\left(\frac{u^{2}+w^{2}}{2}+g z\right)=0 .
$$

\section{b. The separation condition}

The onset or inhibition of separation is related to presence or otherwise of deceleration of the flow parallel to and just outside the viscous boundary layer (e.g., Landau and Lifschitz 1987). For incompressible unstratified flows without external body forces this is equivalent to a condition on the streamwise pressure gradient, as a result of Bernoulli's theorem. However, for the more general stratified case considered here this equivalence is lost, and one has to consider the actual deceleration or acceleration of the flow just outside the inner boundary layer.

Following the approach of Marshall and Tansley (2001), Eq. (7) is integrated over the area, ABCD, sketched in Fig. 1. The area is bounded by two streamlines, one at the outer edge of the inner boundary layer that envelopes the orography $(\mathrm{AB})$ and the other some-

\footnotetext{
${ }^{1}$ Note that $\zeta$ as defined here is the negative of the $y$ component of the three-dimensional vorticity, in order to emphasize the analogy with the $\beta$-plane problem.
} 


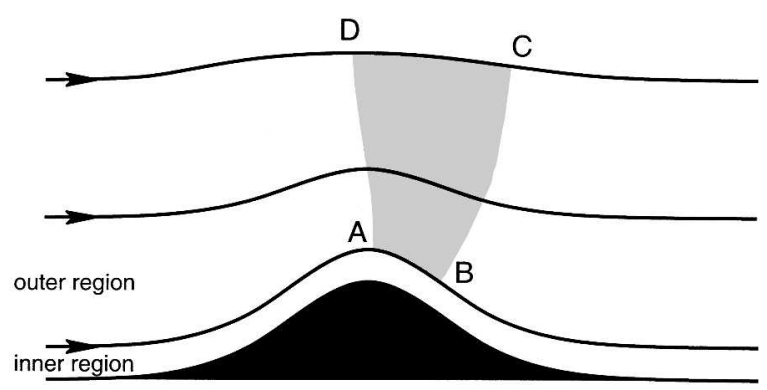

FIG. 1. Schematic of the integration area (shaded) used in the derivation of the vorticity budget in natural coordinates [Eq. (8)]. Curves $\mathrm{AB}$ and $\mathrm{DC}$ are streamlines; curves $\mathrm{BC}$ and $\mathrm{AD}$ are perpendicular to streamlines. Streamline AB lies just outside the inner boundary layer.

where in the outer region (DC), and by two contours normal to streamlines (AD and $\mathrm{BC}$ ).

In evaluating the integral natural coordinates $(s, n)$ are adopted, where $\mathbf{s}$ is the unit vector tangent to the streamlines and $\mathbf{n}$ is the unit vector normal to streamlines [by convention $\mathbf{n}$ points to the left of $\mathbf{s}$; see Dutton (1995) for further details]. In this natural coordinate system the velocity is $u \mathbf{s}$, with $v^{2}=u^{2}+w^{2}$, and the vorticity is given as

$$
\zeta=\frac{v}{R}-v_{n}
$$

where $R$ is the radius of curvature of the streamlines (with positive values denoting deflection to the left).

After making use of Gauss' theorem, the integrated vorticity budget can be written as

$$
\left[\frac{v^{2}}{2}\right]_{\mathrm{A}}^{\mathrm{B}}-\left[\frac{v^{2}}{2}\right]_{\mathrm{D}}^{\mathrm{C}}=-\left[\int \frac{v^{2}}{R} d n\right]_{\mathrm{AD}}^{\mathrm{BC}}-\iint_{\mathrm{ABCD}} B^{*} v d A,
$$

with $[f]_{Q}^{P} \equiv f_{P}-f_{Q}$ and

$$
B^{*} v=\frac{N^{2}}{g u} \frac{\partial}{\partial s}\left(\frac{v^{2}}{2}+g z\right) v
$$

According to Eq. (8) the difference in acceleration between $\mathrm{AB}$ and $\mathrm{DC}$ depends on two processes: a streamline curvature effect and a buoyancy effect. The former results from transfer between shear vorticity and curvature vorticity and may be viewed as a kinematic effect dependent on the geometry of the streamlines. The term involving $B^{*}$ is proportional to the buoyancy frequency, so is only present in stratified fluids.

For hills higher than, say, $100 \mathrm{~m}$ and velocities of the order of $10 \mathrm{~m} \mathrm{~s}^{-1}$ the variations in $g z$ dominate the variations in $v^{2} / 2$ in $B^{*}$. So in this limit

$$
B^{*} v \approx \frac{N^{2}}{u} \frac{\partial z}{\partial s} v=\frac{N^{2} w}{u} .
$$

This is analogous to the advection of planetary vorticity in the oceanic vorticity budget (Marshall and Tansley 2001). The analogy between steady stratified flow over a hill and steady horizontal flow with varying Coriolis parameter was pointed out by Long (1953). Here, the analog of $\beta$, the gradient of the Coriolis parameter, is $N^{2} / u$. Indeed, a stationary gravity wave in a uniform background flow $U$ has total wavenumber $K=N / U$, which may be written as $(\beta / U)^{1 / 2}$, the total wavenumber of a stationary Rossby wave on a $\beta$ plane.

The term involving $B^{*}$ in the separation condition, Eq. (8), has a straightforward physical interpretation, illustrated schematically in Fig. 2. Upstream of the hill, the potential temperature surfaces are inclined upward in the downstream direction so that the baroclinic production term is a source of negative vorticity [according to Eq. (10) the sign of $B^{*} v$ is the same as that of $w$, assuming $u>0$ ]. This source will decelerate the flow close to the surface relative to the flow aloft. The reverse is true when the potential temperature surfaces are inclined downward.

\section{c. Inclusion of mechanical and buoyancy forcing}

For completeness, the vorticity budget in the presence of a mechanical forcing, $\mathbf{F}=(F, G)$, and heating, $H$, is

$$
\begin{aligned}
\nabla \cdot(\zeta \mathbf{u}) & +\frac{N^{2}}{g u} \mathbf{u} \cdot \nabla\left(\frac{u^{2}+w^{2}}{2}+g z\right)=-\frac{H p_{z}}{\rho u \theta}+G_{x} \\
-F_{z} & +\frac{N^{2} \mathbf{u} \cdot \mathbf{F}}{g u} .
\end{aligned}
$$

The effect of heating in this equation is twofold: first, it is represented in the changed locations of the steady

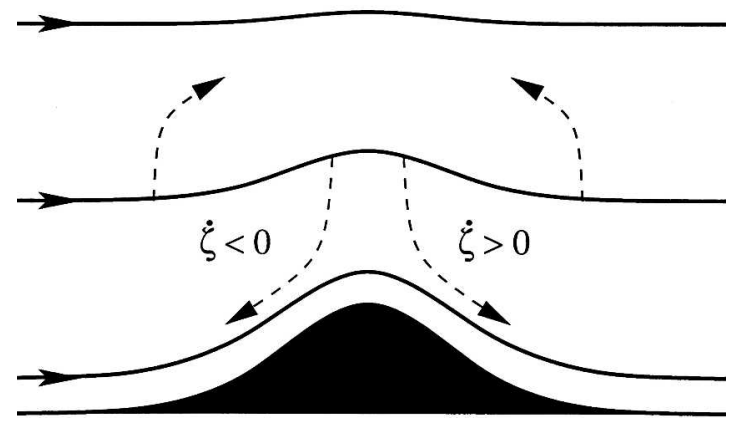

FIG. 2. Schematic illustrating the role of baroclinic production of vorticity $(\dot{\zeta})$ on decelerating the flow on the approach to the hill and accelerating the flow in the lee of the hill (relative to the flow aloft). 
streamlines. Second, the term with $H$ in the numerator compensates for the fact that streamlines and lines of constant potential temperature are no longer coincident. The two combine to give the tilt of the potential temperature surfaces relative to the pressure surfaces. In general, positive heating will rotate lines of constant potential temperature compared to the streamlines in a clockwise fashion that leads to a positive tendency in the vorticity budget. In the upslope region this then reduces the deceleration along the boundary layer; in the downslope region it enhances the acceleration. On both sides diabatic heating inhibits separation. The effect of mechanical friction is opposite: it is safe to assume that $\mathbf{u} \cdot \mathbf{F}<0$ in which case the flow is decelerated everywhere. The consequent facilitation of separation was, for example, found in numerical experiments of Doyle and Durran (2002).

\section{Asymptotic regimes}

\section{a. Hills of small aspect ratio}

It is illuminating to consider the linearized version of the separation formula, Eq. (8), in which the basic-state velocity $U$ is assumed independent of height. Assume that the hill has a very low aspect ratio $h / L$, with $h$ the maximum height of the hill and $L$ the half-width. Perturbation velocities $\left(u^{\prime}, w^{\prime}\right)$ will then be of order $O(h / L)$ compared to the basic-state velocity $U$.

The three terms in the separation formula can then be linearized as follows. First,

$$
\left[\frac{v^{2}}{2}\right]_{\mathrm{A}}^{\mathrm{B}}-\left[\frac{v^{2}}{2}\right]_{\mathrm{D}}^{\mathrm{C}}=U\left(\left[u^{\prime}\right]_{\mathrm{A}}^{\mathrm{B}}-\left[u^{\prime}\right]_{\mathrm{D}}^{\mathrm{C}}\right)+O\left(u^{\prime 2}, w^{\prime 2}\right) .
$$

Now introduce the field $z^{\prime}(x, z)$ describing the vertical departure of the streamlines from their unperturbed upstream equilibrium position. In the linear approximation the curvature $R$ of the streamlines is related to $z^{\prime}$ as $1 / R=z_{x x}^{\prime}$. This then leads to the following linearization for the second term in the separation formula:

$$
\left[\int \frac{v^{2}}{R} d n\right]_{\mathrm{AD}}^{\mathrm{BC}}=U^{2}\left[\int z_{x x}^{\prime} d z\right]_{\mathrm{AD}}^{\mathrm{BC}}+O\left(u^{\prime 2}, w^{\prime 2}\right),
$$

where in the linear approximation, $d n=d z$ and $\left(u^{\prime}\right.$, $\left.w^{\prime}\right) / U=O\left(z_{x}^{\prime}\right)$. Finally,

$$
\begin{aligned}
\iint_{\mathrm{ABCD}} B^{*} v d A= & \iint_{\mathrm{ABCD}}\left(\frac{N^{2}}{g} U u_{x}^{\prime}+N^{2} z_{x}^{\prime}\right) d x d z \\
& +O\left(u^{\prime 2}, w^{\prime 2}\right) .
\end{aligned}
$$

The ratio of the two contributing terms, using $u^{\prime} / U=$ $O\left(z_{x}^{\prime}\right)$ equals $U^{2} / g L_{u}$, with $L_{u}=u^{\prime} / u_{x}^{\prime} \sim L$. A similar scaling as in Eq. (10) can be used to deduce that this is generally a small number and the first contribution can therefore be ignored. With these approximations and using Green's integration theorem

$$
\begin{aligned}
\iint_{\mathrm{ABCD}} B^{*} v d A & \approx \iint_{\mathrm{ABCD}} N^{2} z_{x}^{\prime} d x d z \\
& =\oint_{\mathrm{ABCD}} N^{2} z^{\prime} d z \\
& =\left[\int N^{2} z^{\prime} d z\right]_{\mathrm{AD}}^{\mathrm{BC}},
\end{aligned}
$$

where it is assumed that $N^{2}$ is constant over area $\mathrm{ABCD}$. Now collecting all terms we arrive at

$$
\left[\frac{u^{\prime}}{U}\right]_{\mathrm{A}}^{\mathrm{B}}-\left[\frac{u^{\prime}}{U}\right]_{\mathrm{D}}^{\mathrm{C}} \approx-\left[\int\left(z_{x x}^{\prime}+\frac{N^{2}}{U^{2}} z^{\prime}\right) d z\right]_{\mathrm{AD}}^{\mathrm{BC}} .
$$

The limit for an infinitesimally shallow integration domain, $\left[\left(z_{C}-z_{B}\right) \sim\left(z_{D}-z_{A}\right) \rightarrow 0\right]$ gives a formula for the vertical gradient of $u^{\prime}$. Assuming all fields are continuous, we find for this limit,

$$
\left[\frac{1}{U} \frac{\partial u^{\prime}}{\partial z}\right]_{\mathrm{A}}^{\mathrm{B}} \approx\left[z_{x x}^{\prime}+\frac{N^{2}}{U^{2}} z^{\prime}\right]_{\mathrm{A}}^{\mathrm{B}},
$$

where the fields are to be evaluated on the streamline on which $\mathrm{A}$ and $\mathrm{B}$ lie.

Now consider a prescribed corrugated topography with a single horizontal wavenumber $k=\pi / 2 L$ and amplitude $h / 2$, that is, $z^{\prime}(x, 0)=(h / 2) \cos (k x)$-see Fig. 3 . Linear gravity waves on a constant background velocity satisfy

$$
k^{2}+m^{2}=(N / U)^{2}
$$

(e.g., Baines 1995). Here, $m$ is the vertical wavenumber, which is imaginary when $k>N / U$. For this corrugated topography Eq. (12) can be rewritten as

$$
\left[\frac{u^{\prime}}{U}\right]_{\mathrm{A}}^{\mathrm{B}}-\left[\frac{u^{\prime}}{U}\right]_{\mathrm{D}}^{\mathrm{C}}=-\left[\int m^{2} z^{\prime} d z\right]_{\mathrm{AD}}^{\mathrm{BC}},
$$

and Eq. (13) can be rewritten as

$$
\left[\frac{1}{U} \frac{\partial u^{\prime}}{\partial z}\right]_{\mathrm{A}}^{\mathrm{B}}=\left[m^{2} z^{\prime}\right]_{\mathrm{A}}^{\mathrm{B}} .
$$


(a)

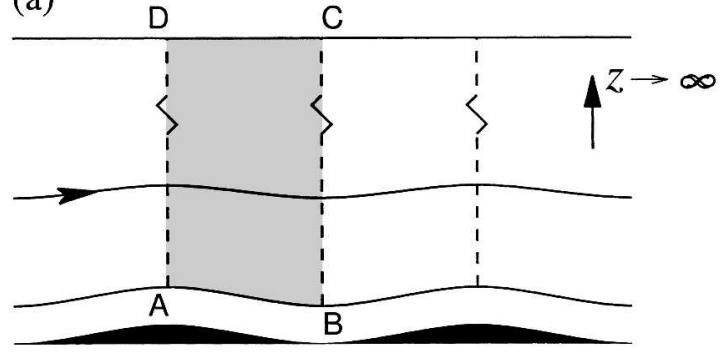

(b)

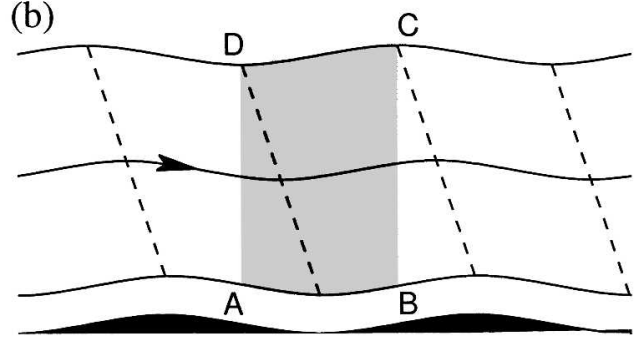

FIG. 3. Integration areas (shaded) used in the linearized Eqs. (14) and (15) for (a) evanescent solutions and for (b) wavelike solutions. The boundaries $\mathrm{AD}$ and $\mathrm{BC}$ are vertical in the linear limit. Also shown are the phase lines (dashed).

We need to distinguish the cases where $m^{2}<0$ (evanescent solutions) and where $m^{2}>0$ (wavelike solutions).

\section{1) Evanescent solutions $\left(m^{2}<0\right)$}

In this case we choose streamline DC infinitely far above the hill so that velocity changes along DC vanish (Fig. 3a). The $z^{\prime}$ field satisfies

$$
z^{\prime}(x, z)=z^{\prime}(x, 0) \exp (-\lambda z) ; \quad \lambda^{2}=-m^{2} ; \quad \lambda>0 .
$$

Substituting in Eq. (14) then gives

$$
\left[\frac{u^{\prime}}{U}\right]_{\mathrm{A}}^{\mathrm{B}}=\lambda\left[z^{\prime}\left(x_{B}, 0\right)-z^{\prime}\left(x_{A}, 0\right)\right] .
$$

So as long as the slope of the topography is negative $\left[z^{\prime}\left(x_{B}, 0\right)<z^{\prime}\left(x_{A}, 0\right)\right]$ the flow will decelerate. The minimum velocity is therefore reached in the trough of the hills. Substituting the prescribed topography above, the maximum achievable deceleration between the top of the hill and the bottom is

$$
\left[\frac{u^{\prime}}{U}\right]_{\mathrm{A}}^{\mathrm{B}}=-\left[\left(\frac{\pi h}{2 L}\right)^{2}-\left(\frac{N h}{U}\right)^{2}\right]^{1 / 2} .
$$

If this deceleration is large, say of $O(1)$, separation will occur. Because this deceleration is realized on the leeside of the hill, this is where the separation will occur.

\section{2) Wavelike SOlutions $\left(m^{2}>0\right)$}

As in this case all fields are periodic functions of $x$ and $z$, we can integrate Eq. (15) along a streamline to find $^{2}$

$$
\partial u^{\prime} / \partial z=m^{2} U z^{\prime} .
$$

Because both $u^{\prime}$ and $z^{\prime}$ are periodic with the same wavenumbers, $u^{\prime}$ is a maximum where $z^{\prime}=0$, and vice versa. So the net acceleration between two points on the same streamline equidistant from an inflection point of $z^{\prime}$ (i.e., where $z^{\prime}=0$ ) will vanish. In the choice of integration domain sketched in Fig. 3b, the net acceleration along DC vanishes. The acceleration along $\mathrm{AB}$ therefore is

$$
\begin{aligned}
{\left[\frac{u^{\prime}}{U}\right]_{\mathrm{A}}^{\mathrm{B}} } & =-\left[\int m^{2} z^{\prime} d z\right]_{\mathrm{AD}}^{\mathrm{BC}} \\
& =-h m^{2} \int_{0}^{\pi / 2 m} \sin (m z) d z \\
& =-h m=-\left[\left(\frac{N h}{U}\right)^{2}-\left(\frac{\pi h}{2 L}\right)^{2}\right]^{1 / 2} .
\end{aligned}
$$

Again, separation will occur if this deceleration is sufficiently large, of $O(1)$. The deceleration now occurs in the trough behind the hill. The flow has decelerated most $3 / 4$ of a wavelength downstream of the peak, so the separation is most likely to occur there first and may therefore be thought of as postwave separation. Because of the periodicity of the hills, this postwave separation is equivalent to a blocked upstream region ahead of the hill. Note that the separation immediately behind the peak of the hill is suppressed because the flow accelerates over the peak.

Figure 4 is the linear phase diagram showing the maximum reduction in $u^{\prime} / U$ as a function of $h / L$ and $N h / U$. The axes are chosen to correspond to the experimentally obtained phase diagram Fig. 5 of Baines (1995). It is assumed that separation occurs when the maximum deceleration becomes comparable to the basic-state velocity. The isoline in the linear phase diagram corresponds therefore to maximum reductions in $u^{\prime} / U$ of $O(1)$, above which separation is supposed to occur. Note that at these lines the linear approximation breaks down. Similarly, the linear phase diagram is strictly only valid where $h / L \ll 1$, that is, the lower part of Fig. 4. Notwithstanding these caveats, the linear

\footnotetext{
${ }^{2}$ One of the reviewers pointed out that this relationship can also be derived from the kinetic relation for linear flow: $w^{\prime}=U z_{x}^{\prime}$. Since the flow is nondivergent $u^{\prime}=-U z_{z}^{\prime}$, and thus $u_{z}^{\prime}=U z_{z z}^{\prime}$, which yields the present result after assuming wavelike perturbations with vertical wavenumber $m$.
} 


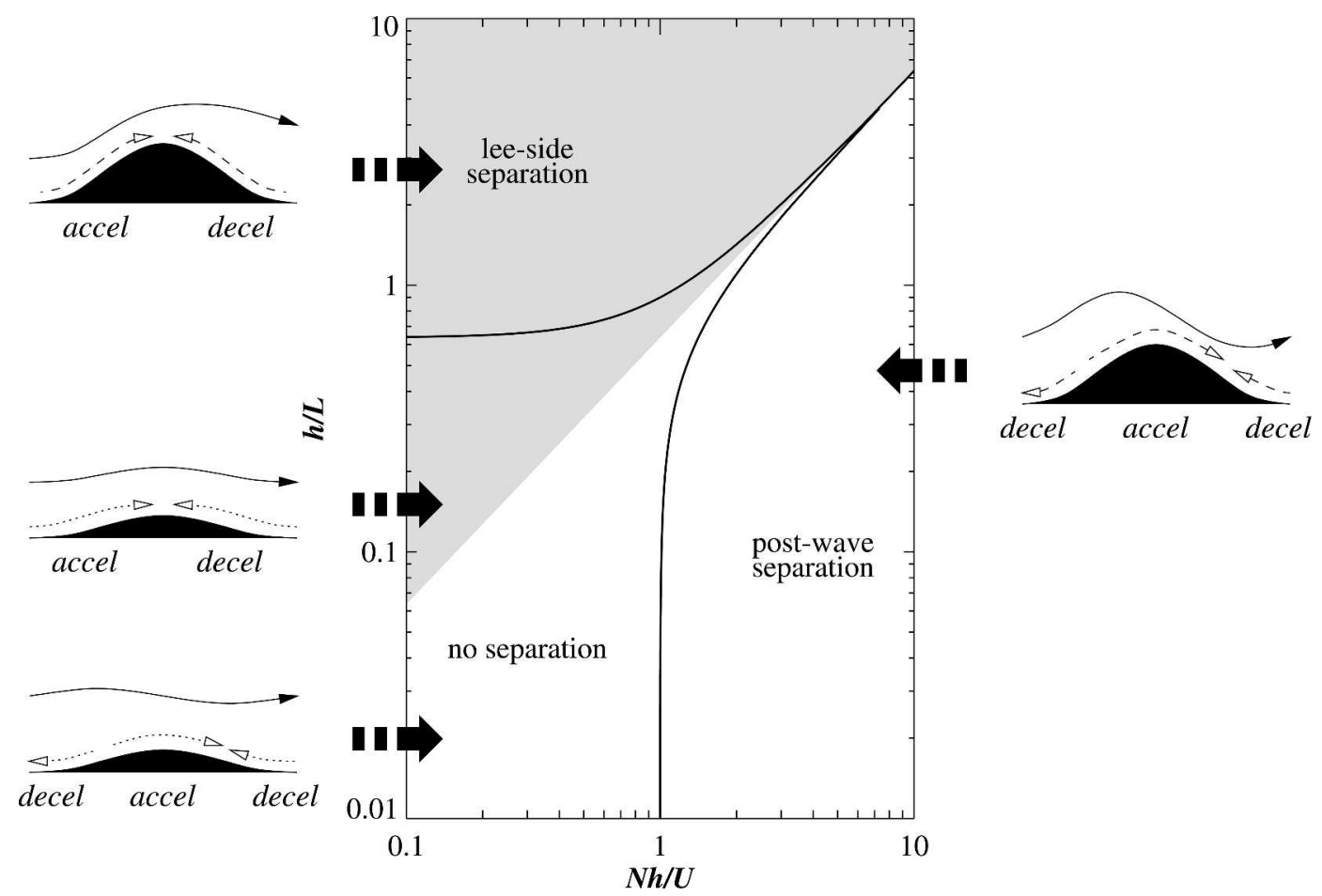

FIG. 4. Phase diagram for the linear case as a function of parameters $h / L$ and $N h / U$. The shaded part of the diagram corresponds to the evanescent regime, the rest of the diagram to the wavelike regime. The two phase lines correspond to horizontal velocity changes with a magnitude equal to the basic-state velocity.

theory predicts the experimentally obtained phase boundaries of Fig. 5 remarkably well.

For both the evanescent and wavelike regimes, it turns out that imbalance between the curvature and the stratification contributions to the vorticity budget leads to appreciable decelerations and separation somewhere at the surface. In general, separation is governed by a combination of hill aspect ratio $h / L$ and stratification $N h / U$. However, for low $N h / U$, leeside separation will occur when $h / L$ exceeds a critical value. For low $h / L$ postwave separation will occur when $N h / U$ exceeds a critical value.

\section{b. Hills of $O(1)$ aspect ratio}

Next consider the case of very strong curvature, where the slope of the attached streamlines is of order unity and perturbation velocities are of the same order of magnitude as the inflow velocity. The geometry of the streamlines will in general be too complicated to evaluate Eq. (8). However, assuming that there is only one length scale for the attached flow, namely the radius of curvature $r$ of the orographic feature, this may be used to find scalings for the terms in the separation formula.

Assume again that in Eq. (8), the term $B^{*} v$ is domi- nated by $N^{2} w / u$. Assuming that in this regime the slopes will be of order unity, this term scales as $N^{2}$. As the radius of curvature is the only length scale for the attached flow, the geometric area scales as $r^{2}$. Likewise, the streamwise change in the term $v^{2} / R$ scales with $U^{2} / r$ and the integration interval scales with $r$. As before, if these two terms are strongly imbalanced, separation will occur. If the curvature term dominates the stratification term, leeside separation will occur. Using the above scalings this corresponds to

$$
r<U / N=\left(U / B^{*}\right)^{1 / 2}
$$

which is analogous to the condition found in Marshall and Tansley (2001).

It is used here that in Eq. (9) the second contribution to $B^{*} v$ dominates. The ratio of the first to the second contribution to $B^{*} v$ scales as $U^{2} / g r$, which for very small radii of curvature $\left(r<U^{2} / g\right)$ can be large. A similar calculation as above then shows that for such cases leeside separation will occur when $r<g / N^{2}$. But for typical atmospheric parameters this inequality is always satisfied, because $r$ is assumed to be smaller than $U^{2} / g$. It must be concluded that for very small curvatures, such that $r<U^{2} / g$, separation will always occur, independent of the stratification. 


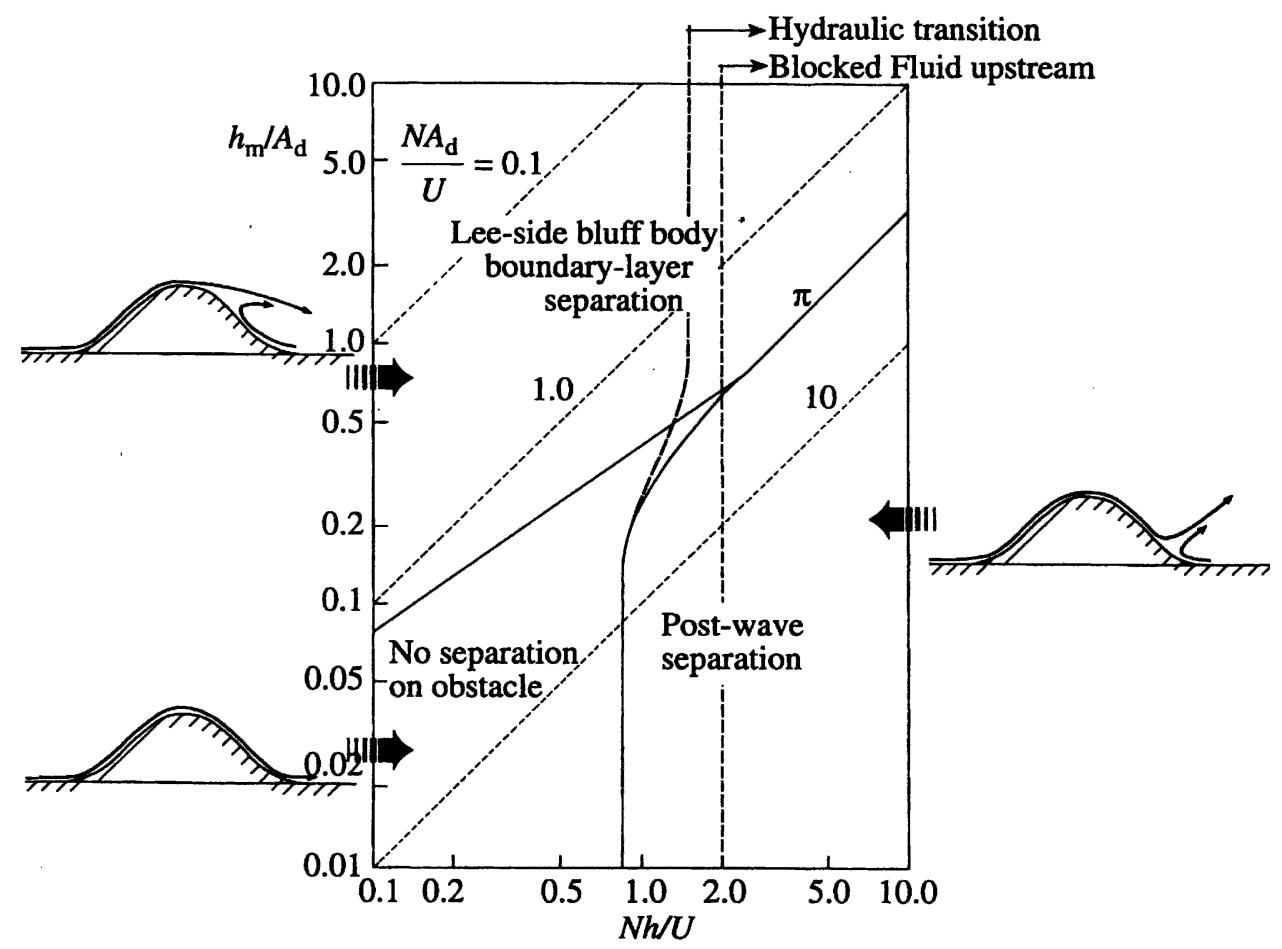

FIG. 5. Reproduction of Fig. 5.8 from Baines (1995). Experimentally found separation regimes as a function of $h_{m} / A_{d}$ and $N h / U$, where $h=h_{m}$ is the maximum height of the hill and $A_{d}$ is the downstream half-width of the hill.

\section{Concluding remarks}

Separation of stratified flow over a two-dimensional hill is inhibited or facilitated by acceleration or deceleration of the flow just outside the attached boundary layer. In this note, a vorticity budget in natural coordinates has been derived and used to find an expression, Eq. (8), for this acceleration or deceleration in terms of streamline curvature and stratification. This formula is used to derive a full regime diagram (Fig. 4) in the case of linear perturbations valid for vanishing hill aspect ratio. In the case of $O(1)$ hill aspect ratio scaling arguments can be used to evaluate the terms in the formula. It is found that separation occurs when a critical aspect ratio is exceeded and for very high aspect ratios separation is expected to occur irrespective of the strength of the stratification. Additionally, diabatic and frictional effects have been considered in the present framework, indicating that diabatic heating inhibits separation everywhere whereas friction facilitates separation.

Figure 5, reproduced from Baines (1995), summarizes flow regimes as a function of $N h / U$ and aspect ratio $h / A$ as found in laboratory experiments by Baines and Hoinka (1985). It is seen that occurrence of leeside separation is a function of $N A / U$, where $A$ is the down- stream half-width of the hill. This relationship holds even after a hydraulic transition has occurred. The present theory can also be applied in this regime. Similar results are shown in numerical experiments by Bacmeister and Pierrehumbert (1988), where it is seen that for high $N h / U$ a hydraulic transition occurs, but as long as the hill is wide enough (in their case $N A / U \approx 10$ ) no leeside separation is observed. In Fig. 5 it is also seen that the region of postwave separation corresponds to large $N A / U$. In the linear theory this regime corresponds to enhanced upstream separation. Indeed it is experimentally found that postwave separation is usually associated with a blocked region ahead of the hill, which may be interpreted as upstream flow separation.

In addition to these experiments, our results are consistent with a wide range of observations. Field observations by Scorer (1955) describe how leeside eddies at a hill occur most often when air is well mixed up to great height. The well-mixed air is an indication of low stratification, which according to our theory indeed favors leeside separation. In the same paper, observations are described where convective stirring at the windward side of the hill inhibits separation there, again consistent with our theory. Not only is the convective stirring associated with reduced stratification, it can also be associated with diabatic heating ahead of the hill, which 
according to our theory also should inhibit windward separation. It is also established that for stable nighttime conditions air in valleys separates from the air aloft (e.g., Mason 1987). From our linear theory it follows that stable stratification inhibits separation immediately behind the peak of the hill, while upstream separation will be stimulated. The separation of air in a valley may then be associated with a blocked region ahead of the downstream hill.

The application of two-dimensional nonviscous steady theory to real flow over hills has its obvious limitations, such as the neglect of three-dimensional effects, turbulence, or Coriolis forces. However, it is likely that the basic mechanisms for the accelerations and decelerations are the same in the more realistic situations, and our results may be applicable to these more general cases.

Acknowledgments. We thank Stephen Belcher for helpful discussions. Two anonymous reviewers are thanked for their insightful comments on the original manuscript that, among other things, stimulated us to significantly expand the linear analysis in section 3 . This work was funded by the U.K. Natural Environment Research Council through the COAPEC thematic programme (NER/T/S/2002/00310).

\section{REFERENCES}

Bacmeister, J. T., and R. T. Pierrehumbert, 1988: On high-drag states of nonlinear stratified flows over an obstacle. J. Atmos. Sci., 45, 63-80.

Baines, P. G., 1995: Topographic Effects in Stratified Flows. Cambridge University Press, 482 pp.

- and K. P. Hoinka, 1985: Stratified flow over two-dimensional topography in fluid of infinite depth: A laboratory simulation. J. Atmos. Sci., 42, 1614-1630.
Belcher, S. E., and N. Wood, 1996: Form and wave drag due to stably stratified flow over low ridges. Quart. J. Roy. Meteor. Soc., 122, 863-902.

Boyer, D. L., and P. A. Davies, 1982: Flow past a circular cylinder on a $\beta$-plane. Philos. Trans. Roy. Soc. London, 306A, 533556.

Doyle, J., and D. R. Durran, 2002: The dynamics of mountainwave-induced rotors. J. Atmos. Sci., 59, 186-201.

Dutton, J. A., 1995: Dynamics of Atmospheric Motion. Dover, 617 pp.

Foster, M. R., 1985: Delayed separation in eastward, rotating flow on a $\beta$-plane. J. Fluid Mech., 155, 59-75.

Goldstein, S., 1948: On laminar boundary layer flow near a point of separation. Quart. J. Mech. Appl. Math., 1, 43-69.

Holden, J. J., S. H. Derbyshire, and S. E. Belcher, 2000: Tethered balloon observations of the nocturnal stable boundary layer in a valley. Bound.-Layer Meteor., 97, 1-24.

Landau, L. D., and E. M. Lifschitz, 1987: Fluid Mechanics. Vol. 6, Course of Theoretical Physics, 2d ed. Pergamon Press, 539 pp.

Long, R. R., 1953: Some aspects of the flow of stratified fluids. I. A theoretical investigation. Tellus, 5, 42-58.

Marshall, D. P., and C. E. Tansley, 2001: An implicit formula for boundary current separation. J. Phys. Oceanogr., 31, 16331638.

Mason, P. J., 1987: Diurnal variations in flow over a succession of ridges and valleys. Quart. J. Roy. Meteor. Soc., 113, 11171140.

Merkine, L. O., 1980: Flow separation on a $\beta$-plane. J. Fluid Mech., 99, 399-409.

Page, M. A., and E. R. Johnson, 1990: Flow past cylindrical obstacles on a beta-plane. J. Fluid Mech., 221, 349-382.

Scorer, R. S., 1955: The theory of airflow over mountains: IVSeparation of flow from the surface. Quart. J. Roy. Meteor. Soc., 81, 340-350.

Stewartson, K., 1970: Is the singularity at separation removable? J. Fluid Mech., 44, 347-364.

Tansley, C. E., and D. P. Marshall, 2001: Flow past a cylinder on a $\beta$ plane, with application to Gulf Stream separation and the Antarctic Circumpolar Current. J. Phys. Oceanogr., 31, 32743283. 